\title{
Parcela ótima para a cultura do cafeeiro obtido por simulação de dados com variâncias conhecidas
}

\author{
Juracy Mendes Moreira ${ }^{1 *}$, Aurélio Ferreira Melo ${ }^{2}$, Jose Marcelo de Oliveira ${ }^{3}$, Daniela
} Silva Ataides ${ }^{4}$, Marcelo Carlos Ribeiro ${ }^{5}$, Juliano Bortolini ${ }^{6}$

${ }^{I}$ Prof. Ms. Faculdade Almeida Rodrigues (FAR), Rio Verde - Goiás, Brasil. E-mail: juracimendesmoreira@yahoo.com.br

${ }^{2}$ Prof. Doutorando Faculdade Almeida Rodrigues (FAR), Rio Verde - Goiás, Brasil. E-mail: aurelioferreiramelo1@hotmail.com

${ }^{3}$ Prof. Ms. Faculdade Almeida Rodrigues (FAR), Rio Verde - Goiás, Brasil. E-mail: oliveirademarcelo@hotmail.com

${ }^{4}$ Profa. Esp. Faculdade Almeida Rodrigues (FAR), Rio Verde - Goiás, Brasil.E-mail: danisila@uol.com.br

${ }^{5}$ Doutorando em Estatística Aplicada e Biometria. UFV. Minas Gerais, Brasil.E-mail:marcelocarlosribeiro@hotmail.com

${ }^{6}$ Prof. Dr. Departamento de Estatística, Universidade Federal de Mato Grosso - Cuiabá, Brasil. E-mail: julianobortolini@ufmt.br

*Autor para correspondência

\begin{abstract}
RESUMO. Diversos métodos têm sido empregados por pesquisadores para determinar o tamanho ótimo de parcela experimental e o controle do erro experimental. Os mais difundidos são: método empírico de Smith; método da inspeção visual da curvatura máxima, método da curvatura máxima do coeficiente de variação. Recentemente foram propostos métodos do ajuste de modelo segmentado (linear com platô) para determinação do tamanho ótimo de parcela. Todos os métodos citados anteriormente utilizam dados provenientes de ensaios de uniformidade para medir a variabilidade entre os possíveis tamanhos de parcela. Os dados utilizados neste trabalho foram obtidos através de simulação computacional de dados de um ensaio de uniformidade. O objetivo proposto neste trabalho consiste em utilizar técnicas estatísticas para determinar o tamanho ótimo de amostra. Conclui-se o tamanho ótimo de parcela foi conseguido através do método de máxima curvatura do coeficiente de variação que apresentou um $\mathrm{R}^{2}$ de 0,9818 .
\end{abstract}

Palavras chave: Métodos, simulação, uniformidade, tamanho de parcela

\section{Optimum plot for coffee culture obtained by data simulation with known variances}

\begin{abstract}
Several methods have been used by researchers to determine the optimum size of experimental plot and control of experimental error. The most widespread are: empirical method of Smith, method of visual inspection of maximum curvature, method of maximum curvature coefficient of variation. Some methods of segmented model adjustment was recently proposed to (linear with plateau) to determine the optimum plot size. All methods mentioned above have used data from assays for measuring uniformity variability between possible plot sizes. The data used in this study were obtained through computer simulation data of a uniformity test. The goal proposed in this paper is to use statistical techniques to determine the optimal sample size. We conclude the optimum plot size was achieved by the method of maximum curvature coefficient of variation which showed a R2 of 0.9818 .
\end{abstract}

Keywords: Methods, simulation, uniformity and plot size

\section{Introdução}

O método empírico de Smith desenvolvido por Smith (1938) com base no índice de heterogeneidade do solo $(b)$, que é obtido de uma relação empírica entre a variância da parcela e o tamanho da parcela, esse método é conhecido como a lei empírica de Smith e foi a precursora de vários outros métodos usados na determinação do tamanho ótimo de parcelas estabelecendo uma relação entre a variância e o tamanho da parcela. 
A relação de Smith é dada por:

$$
V U_{(x)}=\frac{V_{1}}{X^{b}}
$$

em que: $V U_{(\mathrm{x})}$ a variância por unidade calculada entre as parcelas de $X$ unidades básicas; $V_{1}$ é a variância dos valores de parcelas constituídos de uma unidade básica; $b$ é o índice de heterogeneidade do solo pertencente ao intervalo $(0 \leq b \leq 1)$ e $X$ é o número de unidades básicas que compõem a parcela.

$\mathrm{O}$ índice de heterogeneidade do solo $(b)$ indica o grau de correlação entre parcelas experimentais adjacentes, à medida que se aproxima da unidade menor será a correlação entre as parcelas adjacentes (alta heterogeneidade), entretanto, quando se aproxima do zero maior será a correlação entre as parcelas (baixa heterogeneidade). $O$ índice de heterogeneidade do solo " $b$ ", de Smith pode ser obtido através da linearização da equação mediante uma transformação logarítmica, em que $\log V U_{(x)}=\log V_{1}-b \times \log X$. Sendo assim, " $b$ " poderá ser estimado como um coeficiente angular de regressão linear. Utilizando o método de Smith para a determinação do índice de heterogeneidade do solo Feijó et al. (2006) trabalharam com abobrinha italiana cultivada em estufa plástica e encontrou baixo índice de heterogeneidade e tamanho de parcela igual a uma unidade básica.

\section{Tamanho ótimo de parcela}

Cientistas e pesquisadores têm procurado desenvolver métodos mais eficientes na determinação do tamanho ótimo de amostra visando sobre tudo a redução dos custos. As metodologias mais utilizadas são: O método empírico de Smith (1938); o método da inspeção visual da curvatura máxima (Federer, 1955), o método da curvatura máxima do coeficiente de variação (Lessman \& Atkins, 1963) e recentemente foram propostos os métodos de modelos segmentados. Estes métodos utilizam o ajuste de um modelo segmentado (modelo linear com platô) para determinação do tamanho ótimo de parcela (Paranaíba, 2014). Todos os métodos citados anteriormente utilizam dados provenientes de ensaios de uniformidade para medir a variabilidade entre os possíveis tamanhos de parcela.

\section{Método de inspeção visual da curvatura máxima}

Proposto por Federer (1955) para determinar tamanhos ótimos de parcelas, para o uso desse método deve-se implantar e conduzir um ensaio de uniformidade, definir o tamanho de uma unidade básica, $(U B)$ de tamanho $X$, após a colheita das unidades básicas é feito a identificação de cada uma segundo sua posição na linha ou coluna e são feitos agrupamentos entre as unidades básicas adjacentes formando diversos tamanhos de parcelas. Para cada uma dessas parcelas, calculase o coeficiente de variação $\left(C V_{(x)}\right)$. O conjunto de pontos formado pelo par ordenado $\left(X: C V_{(x)}\right)$ são relacionados em um gráfico, cuja união desses pontos fornecerá uma curva (Figura 1). Por meio de inspeção visual determina-se o tamanho ótimo de parcela. O coeficiente de variação é dado por:

$$
C V_{(x)}=\frac{\sqrt{V_{(x)}}}{\bar{y}_{(x)}}
$$

em que, $C V_{(x)}$ é o coeficiente de variação entre parcelas de tamanho $X ; V_{(x)}$ representa a variância total de parcelas em função de $x$ unidades básicas; $\bar{y}_{(x)}$ representa a média.

Segundo Zanon \& Storck (2000) utilizando este método para determinar tamanho ótimo de parcela para Eucaliptos encontram resultados muito discrepante. Em experimentos com mandioca Viana et al. (2002) encontraram tamanhos de parcelas muito grandes.

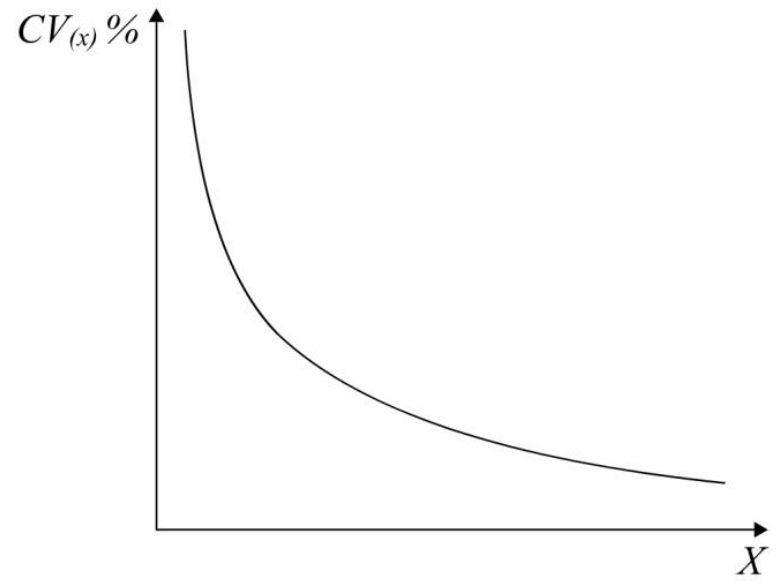

Figura 1. Representação gráfica da relação entre o coeficiente de variação $C V_{(x)}$ e o tamanho de parcela $(X)$.

Método da máxima curvatura do coeficiente de variação

Proposto por (Lessman \& Atkins, 1963), o método de máxima curvatura do coeficiente de variação consiste em uma representação gráfica 
dos coeficientes de variação versus os respectivos tamanhos de parcela. Esse método fornece resultados mais confiáveis, pois estabelece uma regressão para explicar a relação entre os coeficientes de variação e os respectivos tamanhos de parcelas. Essa relação é dada por:

$$
C V_{(x)}=\frac{A}{X^{B}}
$$

em que A e B são obtidos pelo método de mínimos quadrados de Gauss-Newton para modelos não lineares. No gráfico da relação $C V_{(x)}$ versus tamanho de parcela $(X)$, procura-se determinar o ponto correspondente à curvatura máxima do coeficiente de variação por meio de cálculos algébricos (Figura 2).



Figura 2. Tamanho de parcela determinado algebricamente.

Dessa forma o tamanho ótimo de parcela passa a ser determinado algebricamente com o uso da seguinte expressão:

$$
x_{0}=\left[\frac{\hat{A}^{2} \widehat{B}^{2}(2 \hat{B}+1)}{\hat{B}+2}\right]^{\frac{1}{2+2 \widehat{B}}},
$$

em que: $x_{0}$ é o valor da abcissa no ponto de máxima curvatura; $\widehat{A}$ e $\widehat{B}$ os estimadores de $\mathrm{A}$ e $\mathrm{B}$, respectivamente.

Uma das dificuldades de se trabalhar com esses métodos é a necessidade do agrupamento das unidades básicas adjacentes para a obtenção dos diversos tamanhos de parcelas e a determinação dos coeficientes de variação relativa a cada uma destas possibilidades e ainda da dependência de um bom ajuste de um modelo não linear. Um exemplo de utilização deste método no melhoramento da cana de açúcar é apresentado por (Leite et al., 2006) para este experimento foi encontrado tamanhos de parcelas com menos de uma planta, o que é impraticável.

\section{Método do modelo segmentado linear com platô}

Este método foi inicialmente foi usado para determinar a fertilidade de solo. Sua principal vantagem é que com o uso de altas doses de determinado elemento se torna possível estimar a diminuição de desempenho e desenvolvimento ocasionada pelo excesso.

Esse método é constituído por dois segmentos onde para valores de $X_{i} \leq x_{0}$ é descrito por um modelo linear crescente ou decrescente dependendo do valor de $\beta_{1}$ até determinado ponto $P$ que é o platô, e para valores de $X_{i}>x_{0}$ é descrito por uma constante, tal como ilustram as figuras 3 e $\underline{4}$. O ponto de junção dos dois segmentos linear e platô será tomado como tamanho ótimo de parcela. O modelo segmentado linear com platô é dado por:

$Y_{i}=\left\{\begin{array}{lll}\beta_{0}+\beta_{1} X_{i}+\varepsilon_{i} & \text { se } & X_{i} \leq x_{0} \\ P+\varepsilon_{i} & \text { se } & X_{i}>x_{0}\end{array}\right.$,

em que, $Y_{i}$ é a variável resposta ou variável dependente; $\beta_{0}$ é o intercepto do modelo anterior ao platô; $\beta_{1}$ é o coeficiente angular; $P$ é o platô; $x_{0}$ é o ponto de junção dos dois segmentos; $\varepsilon_{i}$ é o erro que está associado a $i$-ésima observação.



Figura 3. Método do modelo segmentado linear com platô.

Este método vem sendo usado em pesquisas envolvendo respostas nutricionais, como exemplo, Portz et al. (2000) utilizaram o ajuste deste modelo para determinar o mínimo de nutrientes exigido em dietas para peixes, visando o máximo de ganho de peso. A proposta de Paranaíba (2014) consiste em ajustar o modelo segmentado linear com platô aos coeficientes de variação obtidos para 
diferentes tamanhos de parcelas em ensaios de uniformidade no contexto de dimensionamento de tamanho ótimo de parcela. Este modelo pode ser definido por:

$$
C V_{(x)}=\left\{\begin{array}{lll}
\beta_{0}+\beta_{1} X_{i}+\varepsilon_{i} & \text { se } X \leq x_{0} \\
C V P+\varepsilon_{i} & \text { se } X>x_{0}
\end{array}\right.
$$

em que, $C V_{(x)}$ é o coeficiente de variação entre os totais para parcelas com $X_{i}$ unidades básicas; $C V P$ é o coeficiente de variação no ponto de junção dos dois segmentos; $\beta_{0}$ é o intercepto do segmento linear; $\beta_{1}$ é o coeficiente angular; $\varepsilon_{i}$ representa um componente aleatório. Pela condição de continuidade os dois segmentos (linear e platô) são iguais e diferenciáveis em $x_{0}$. $\mathrm{E}$ a determinação de $x_{0}$ é feita tomando-se $\beta_{0}+\beta_{1} x_{0}=$ CVP e isolando $x_{0}$, chegando-se à seguinte expressão:

$$
x_{0}=\frac{C V P-\beta_{0}}{\beta_{1}},
$$



Figura 4. Ajuste do modelo linear com platô à curva do $C V_{(x)}$ em função do número de UBs $(X)$ agrupadas.

Método do modelo segmentado quadrática com platô

De maneira análoga ao método anterior este também utiliza dados de um ensaio de uniformidade e é composto por dois segmentos (quadrático e platô), para valores de $X_{i} \leq x_{0}$ é descrito por um modelo quadrático (parábola) e para valores de $X_{i}>x_{0}$ é descrito por uma constante (platô), bem como ilustram as figuras 5 e 6. O ponto de junção dos dois segmentos quadrático e platô será tomado com o tamanho ótimo de parcela e deverá ser estimado. Este modelo pode ser definido por:

$$
Y_{i}=\left\{\begin{array}{ll}
\beta_{0}+\beta_{1} X_{i}+\beta_{2} X^{2}+\varepsilon_{i} & \text { se } X_{i} \leq x_{0} \\
P+\varepsilon_{i} & \text { se } X_{i}>x_{0}
\end{array},\right.
$$

em que, $Y_{i}$ é a variável dependente; $\beta_{0}, \beta_{1} e \beta_{2}$ são os parâmetros do modelo; $x_{0}$ é o tamanho ótimo de parcela; $P$ é o platô; $\varepsilon_{i}$ um componente aleatório.

Este método consiste em ajustar um modelo segmentado quadrático com platô aos coeficientes de variação obtidos para os diferentes tamanhos de parcelas em um ensaio de uniformidade no contexto de dimensionar um tamanho ótimo de parcela. Os dois segmentos linear e quadrático devem ser iguais e diferenciáveis em $x_{0}$. Rezende et al. (2007) utilizaram o ajuste do modelo segmentado quadrática com platô para determinação da exigência nutricional máxima de zinco em frangos de corte. De maneira análoga ao método anterior, o modelo segmentado quadrático com platô ao ser ajustado aos coeficientes de variação pode ser definido pela expressão:

$$
C V_{(x)}=\left\{\begin{array}{lll}
\beta_{0}+\beta_{1} X_{i}+\beta_{2} X^{2}{ }_{i}+\varepsilon_{i} & \text { se } X \leq x_{0} \\
C V P+\varepsilon_{i} & \text { se } X>x_{0}
\end{array},\right.
$$

em que: $C V_{(x)}$ é o coeficiente de variação entre os totais para parcelas com $X_{i}$ unidades básicas; $C V P$ é o coeficiente de variação no ponto de junção dos dois segmentos; $\beta_{0}, \beta_{1}$ e $\beta_{2}$ são os parâmetros de um polinômio do $2^{\circ}$ grau; $x_{0}$ é tomado como o tamanho ótimo de parcela a ser estimado e $\varepsilon_{i}$ representa um componente aleatório sendo $\varepsilon_{1}$, $\varepsilon_{2}, \ldots \varepsilon_{n} \quad$ considerados independentes $\mathrm{e}$ normalmente distribuídos com média zero e variância constante.



Figura 5. Modelo segmentado quadrático com platô

Para garantir a continuidade, os dois segmentos devem ser iguais em $x_{0}$. Assim, resolvendo $\beta_{0}+$ 
$\beta_{1} X_{0}+\beta_{2} X_{0}^{2}=C V P$, isolando $x_{0}$, chega-se à seguinte expressão:

$$
x_{0}=\frac{-\beta_{1}}{2 \beta_{2}},
$$

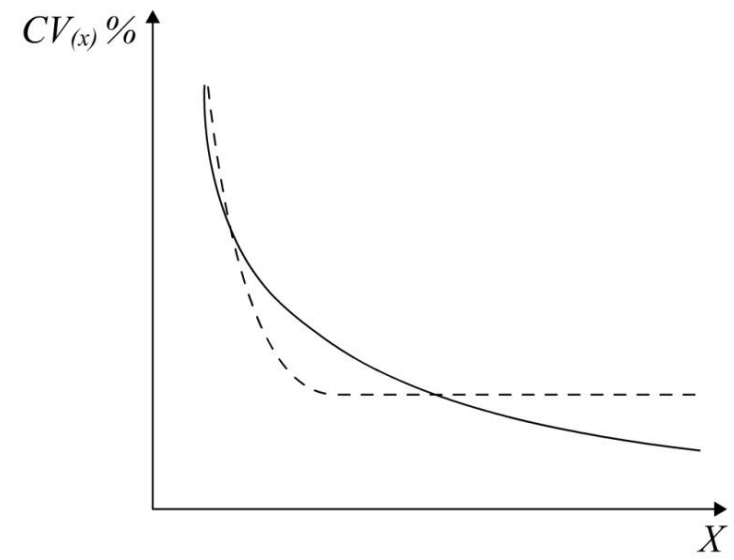

Figura 6. Ajuste do modelo quadrático com platô à curva do $C V_{(x)}$ em função de numero de UB $(X)$ agrupadas.

\section{Avaliadores da qualidade do modelo ajustado}

Como critério para seleção de modelos, partiremos do princípio de que não existe um modelo verdadeiro. Apenas modelos que se aproximam da realidade, havendo com isso perda de informação. O método usualmente conhecido para medir a correlação entre duas variáveis é o coeficiente de correlação linear de Pearson, $\left(R^{2}\right)$, e é uma medida da proporção da variabilidade em uma variável que é explicada pela variabilidade da outra e será tomado como modelo ótimo aquele que apresentar maior $R^{2}$, pode ser obtido pela seguinte expressão:

$$
R^{2}=1-\frac{S Q M_{\text {residuo }}}{S Q_{\text {total }}}
$$

\section{Metodologia}

Os dados utilizados neste trabalho foram obtidos através de simulação computacional de dados, sendo utilizado no processo e simulação uma constante $\mu=100$ e as variâncias (entre) $\sigma_{\alpha}^{2}$ $=0,310807 \mathrm{e}$ (dentro) $\sigma_{\beta}^{2}=0,566276$. Lima (1979). Os dados simulados foram dispostos em uma matriz de grid 90x3. Quando a determinação das unidades amostrada é feita de forma aleatória os parâmetros do modelo, exceto media $\mu$ também têm efeitos aleatórios.
Pelo software estatístico R (Team, 2014) foi possível determinar diversos tamanhos de parcelas. Em seguida esses valores foram agrupados dentro de cada conjunto de dados formando diversos tamanhos de parcelas (sem se importar com a forma desses agrupamentos) e para cada tamanho de parcela foi determinado o valor da estimativa do coeficiente de variação da média; mediana e moda. Moreira (2012) utilizou um estudo de simulação computacional para determinar o tamanho ótimo de amostra biológica para estudo na PCR em tempo real.

Considerando um ensaio de uniformidade formado por um grid $l \times c$ de unidades básicas, com $l$ linhas e $c$ colunas, dispostos do mesmo modo que a figura 7 .

$$
\left[\begin{array}{ccccc}
Z_{11} & Z_{12} & Z_{13} & \cdots & Z_{1 c} \\
Z_{21} & Z_{22} & Z_{23} & \cdots & Z_{2 c} \\
Z_{31} & Z_{32} & Z_{33} & \cdots & Z_{3 c} \\
\vdots & \vdots & \vdots & \ddots & \vdots \\
Z_{r 1} & Z_{r 2} & Z_{r 3} & \cdots & Z_{r c}
\end{array}\right]
$$

Figura 7. Croqui de um ensaio de uniformidade.

As unidades básicas são agrupadas em quantidade $X$ de parcelas de modo que $X$ seja divisor $l \times c$ para cada $X$ de unidades básicas agrupadas são calculados os $C V_{(x)}$, entre as parcelas que compõem o ensaio. Na figura 8 temse um exemplo do agrupamento de duas linhas e uma coluna resultando no tamanho de parcela $X=$ 2 unidades básicas na forma $2 \times 1$.

$$
\left[\begin{array}{cccc}
Z_{11}+Z_{21} & Z_{12}+Z_{22} & \ldots & Z_{1 c}+Z_{2 c} \\
Z_{31}+Z_{41} & Z_{32}+Z_{42} & \ldots & Z_{3 c}+Z_{4 c} \\
\vdots & \vdots & \ddots & \vdots \\
Z_{(r-1) 1}+Z_{r 1} & Z_{(r-1) 2}+Z_{r 2} & \ldots & Z_{(r-1) c}+Z_{r c}
\end{array}\right]
$$

Figura 8. Exemplo ilustrativo de um agrupamento com $X=2$ unidades básicas na forma $2 \times 1$.

\section{Resultado e discussão}

Os valores dos coeficientes de variação para diferentes tamanhos de parcelas encontram-se na tabela 1.

Na tabela 2 estão apresentados os resultados desses agrupamentos e o tamanho ótimo de parcela para cada método citado anteriormente. 
Tabela 1. Coeficientes de variação para diferentes tamanhos de parcelas

\begin{tabular}{lccc}
\hline Parcela & $\begin{array}{c}\text { Media } \\
\text { CV* }\end{array}$ & Mediana CV* & $\begin{array}{c}\text { Moda } \\
\mathrm{CV}^{*}\end{array}$ \\
\hline 1 & 9,341 & 9,335 & 9,308 \\
2 & 6,579 & 6,573 & 6,574 \\
3 & 6,202 & 6,194 & 6,189 \\
6 & 4,957 & 4,952 & 4,939 \\
9 & 3,532 & 3,519 & 3,496 \\
10 & 2,867 & 2,840 & 2,673 \\
18 & 2,812 & 2,789 & 2,750 \\
27 & 2,274 & 2,249 & 2,123 \\
30 & 2,146 & 2,121 & 1,961 \\
45 & 1,212 & 1,181 & 1,131 \\
90 & 0,699 & 0,658 & 0,571 \\
135 & 0,832 & 0,703 & 0,482 \\
\hline
\end{tabular}

*CV Coeficiente de Variação

Tabela 2. Tamanho ótimo de parcela e seus de coeficientes de correlação

\begin{tabular}{lcc}
\hline Modelo & Parcela ótima & Coeficiente de correlação \\
\hline Máxima curvatura do CV & $2,58 \mathrm{UBs}$ & 0,9818 \\
Segmentado linear com platô & $5,22 \mathrm{UBs}$ & 0,8208 \\
Segmentado quadrática com platô & $15,75 \mathrm{UBs}$ & 0.9081 \\
\hline
\end{tabular}

\section{Conclusão}

Conclui-se o tamanho ótimo de parcela foi conseguido através do modelo máxima curvatura do coeficiente de variação que apresentou um tamanho ótimo de parcela de aproximadamente 3 unidades básica com um $\mathrm{R}^{2}$ de 0,9818 .

\section{Referências Bibliográficas}

Federer, W. T. (1955). Experimental design. Mac Millan, New York. 544p.

Feijó, S., L. Storck, A. D. C. Lúcio, and S. J. Lopes. (2006). Heterogeneidade do solo e de tamanho de amostra antes e após cultivos com abobrinha italiana em estufa plástica. Ciencia. Rural 36, 1744-1748.

Leite, D. M. G., Silva, M. A., Medeiros, R. B., Saibro, J. C., Pavan, M. A. \& Barrey, M. A. A. (2006). Efeito de diferentes sistemas de pastejo sobre o desempenho de suínos mantidos em pastagem de trevo-branco (Trifolium repens
L.). Revista Brasileira de Zootecnia. 35, 792796.

Lessman, K. J. \& Atkins, R. E. (1963). Optimum plot size and relative efficiency of lattice designs for grain sorghum yield tests. Crop Science. 3, 477-481.

Lima, P.C. Método de amostragem para a avaliação do índice de infecção da ferrugem do cafeeiro Berk e Br. (1979). Dissertação (Mestrado em Estatística e experimentação agropecuária) - Escola superior de agricultura "Luiz de Queiroz". Piracicaba - SP.

Moreira, J. M., Ferreira, F., Lima, R. R, Lima, A. A. \& Chalfun-Júnior, A. (2012). Tamanho ótimo de amostra biológica para estudo na PCR em tempo real, Revista Brasileira de Biometria, 30, 258-277.

Paranaiba, P. F, Ferreira, D. F. \& Moraes, A. R. (2009). Proposição de métodos para estimar o tamanho ótimo de parcelas experimentais. Revista Brasileira de Biometria, 27, 255. 
Portz, L., Dias, C. T. S., Cyrino, d J. E. P. (2000). Regressão segmentada como modelo na determinação de exigências nutricionais de peixes. Scientia Agricola 57, 601-607.

Rezende, D. M. L. C., Muniz, J. A., Ferreira, D. F. Silva, F. F. \& Aquino, L. H. (2007). Ajuste de modelos de platô de resposta para a exigência de zinco em frangos de corte. Ciência $e$ Agrotecnologia. 31, 468-478.

Smith, H. F. (1938). An empirical law describing heterogeneity in the yields of agricultural crops. Journal Agricultural Science 28, 1-23.

Team, R. C. (2014). R: A language and environment for statistical computing. p 1-14.

Viana, A. E. S., T. Sediyama, P. R. Cecon, S. C. Lopes, and M. A. N. Sediyama. 2002. Estimativas de tamanho de parcela em experimentos com mandioca. Horticultura Brasileira 20, 58-63.

Zanon, M. L. B., and L. Storck. (2000). Tamanho ótimo de parcelas experimentais para Eucalyptus saligna Smith em dois estádios de desenvolvimento. Cerne 6, 104-111.

\section{Article History}

Received 13 May 2016

Accepted 17June 2016

Available on line 25 July 2016

License information: This is an open-access article distributed under the terms of the Creative Commons Attribution License, which permits unrestricted use, distribution, and reproduction in any medium, provided the original work is properly cited. 